\title{
Corrosion of an Implanted Medical Device: Rare-Earth Magnet Case Study
}

\author{
Eric P. Guyer, Bruce G. Pound, Steven Crane
}

Exponent Failure Analysis Associates, Inc., Materials \& Corrosion Engineering

Corrosion is a potential concern with implanted medical devices made of metallic materials. In some cases, the devices contain components with a relatively low corrosion resistance in vivo and so are protected by the use of hermetic casings, coatings, or other approaches. However, despite such protective systems, internal components can undergo corrosion for various reasons. Analysis of the type and cause of the corrosion can provide valuable information in terms of the design and fabrication of other systems requiring use of these materials. In this study, the corrosion of an implanted $\mathrm{NdFeB}$ magnet was investigated after its casing leaked in vivo. The magnet was exposed to physiological fluids, resulting in significant corrosion of the material and an associated change in its magnetic signature. Corrosion rates were determined both on the basis of literature data and from an analysis of explanted magnets. The time to reach a given loss in magnetic field strength was then estimated using a magnetic field strength model coupled with values of the corrosion rate.

\section{Magnetic Field Strength Model}

The magnetic field strength generated by a permanent magnet is a function of several variables including remnant magnetization, magnet dimensions, and measurement distance. In the case of a cylindrical magnet, it can be estimated using the following equation:

$$
\mathrm{B}_{\mathrm{x}}=\frac{\mathrm{B}_{\mathrm{r}}}{2}\left(\frac{(\mathrm{L}+\mathrm{X})}{\sqrt{\mathrm{R}^{2}+(\mathrm{L}+\mathrm{X})^{2}}}-\frac{\mathrm{X}}{\sqrt{\mathrm{R}^{2}+\mathrm{X}^{2}}}\right)
$$

where $B_{x}$ is the measured field, $B_{r}$ is the remnant magnetization, $R$ is the magnet radius, $L$ is the magnet length, and $\mathrm{X}$ is the distance from the magnet surface along the axial centerline. Based on data for the magnet characteristics, the nominal field strength at a distance of $1 \mathrm{~cm}$ away from the magnet was estimated to be $140 \mathrm{G}$, which was consistent with the measured value reported by the device manufacturer for an unexposed unit.

To estimate the time for the field strength to decrease magnetism by $85 \%$ (minimum magnetic strength), the corrosion was assumed to result in metal loss uniformly in a direction radially toward the center. In addition, the material was assumed to become magnetically inactive after being corroded.

\section{Corrosion Rates}

The corrosion rate was determined using two approaches: (1) calculated values based on data in the literature, and (2) empirical estimates using a cross-sectioned explant.

\section{Corrosion Rate from Literature Data}

An electrochemical study showed NdFeB has a corrosion current density of $i=1.53 \times 10^{-8} \mathrm{~A} \mathrm{~cm}^{-2}$ in deaerated Ringer's solution. This value is an apparent current density based on the total surface area of 
the $\mathrm{NdFeB}$ sample. However, $\mathrm{NdFeB}$ is known to undergo intergranular corrosion where the Nd-rich layer intergranular region corrodes preferentially, which can lead to grains of the $\phi$ phase $\left(\mathrm{Nd}_{2} \mathrm{Fe}_{14} \mathrm{~B}\right)$ becoming loose and falling away. Since corrosion is localized at the grain boundaries, the apparent corrosion current density must be corrected to determine the real (or true) corrosion current density.

The correction was made using the ratio of the areas of the $\phi$ grains and the Nd-rich phase at the magnet surface. The grain size of the magnets used was measured from cross-sectioned samples, via lineal grain size analysis and an assumed Nd-rich phase thickness of $3 \mathrm{~nm}$, which is in the middle of the range of reported values. The real current density was estimated and resulted in an estimated corrosion rate $\mathrm{CR}=$ $1.6 \times 10^{-8} \mathrm{~g} \mathrm{~s}^{-1} \mathrm{~cm}^{-2}$. The density of the Nd-rich phase was taken to be the same as the magnet, which results in an estimated corrosion rate of $2.1 \times 10^{-9} \mathrm{~cm} \mathrm{~s}^{-1}$, or $\mathrm{CR}=1.8 \mu \mathrm{m} \mathrm{day}^{-1}$.

\section{Corrosion Rate from Metallography}

In order to image the internal geometry and microstructure after field exposure, explant samples were mounted in epoxy, polished in cross-section, and examined using stereomicroscopy and metallography. The cross-section samples showed an inner region with a microstructure typical of a non-exposed exemplar unit with no corrosion and an outer region with a microstructure exhibiting evidence of intergranular corrosion. The interface separating these regions appeared to run generally parallel to the unit casing, which validated the assumption of a linear corrosion front moving radially inward through significant mesoscopic corrosion pathways.

The metallographic analysis was used to estimate a corrosion rate for comparison with the value obtained using literature data. It was found that the effective corrosion rate $\left(\mathrm{CR}_{\mathrm{eff}}\right)$ could be expressed by Equation 2:

$$
\mathrm{CR}_{\text {eff }}=\frac{1}{2}\left(\frac{\mathrm{D}-\mathrm{D}_{\mathrm{i}}}{\mathrm{t}}\right) .
$$

where $\mathrm{D}$ is the nominal diameter and $\mathrm{D}_{\mathrm{i}}$ is diameter of the intact region. For a $\mathrm{D}$ of $0.84 \mathrm{~cm}$ and $\mathrm{D}_{\mathrm{i}}$ of $0.61 \mathrm{~cm}, \mathrm{CR}_{\text {eff }}$ was estimated to be $1.3 \mu \mathrm{m} /$ day, which is similar to the corrosion rate obtained on the basis of data from the literature.

\section{Time-to-Minimum Magnetic Strength}

The time to reach the minimum magnetic strength was calculated using the corrosion rates obtained from literature data and from metallography. The estimated times were found to be $\sim 4$ years and $\sim 6$ years for corrosion rates of 1.3 and $1.8 \mu \mathrm{m}_{\text {day }}{ }^{-1}$. These time estimates serve as an upper bound for exposed units, since the brittle nature of $\mathrm{NdFeB}$ magnets can lead to macroscopic cracking. Such cracking is difficult to treat using a magnetic model, but it is expected to significantly reduce the time to reach minimum magnetic strength predicted on the basis of the corrosion rates. 